\title{
Ultrasonic Viscoelasticity and Excess Absorption in Polymer Solution
}

\author{
Haruyo YosHIZAKI \\ Department of Materials Science and Engineering, Defense Academy, \\ Hashirimizu 1-10-20, Yokosuka 239, Japan
}

(Received May 12, 1989)

\begin{abstract}
Shear moduli are measured by the reflection method for many polymer-toluene solutions. The results have good agreement with theoretical values by Rouse and Lamb. Longitudinal velocity and absorption are measured for poly(vinylisobuthylether) solution in toluene by the interferometric method. Comparing both moduli, it is found that the ratio of the dynamic volume viscosity to the dynamic shear one is $20-40$. The phenomena are discussed on the basis of several theories and found to be well described by the concentration fluctuation theory by V. P. Romanov and V. A. Solove'ev.

KEY WORDS Shear Impedance / Acoustical Excess Absorption Concentration Fluctuation Theory / Rouse's and Lamb's Theory Poly(vinylisobuthylether) / Polymer Solution /
\end{abstract}

Recently, the relaxation properties of longitudinal waves of relatively dilute solutions in the $100 \mathrm{MHz}$ region have been studied by many scientists, because the rate process for an interaction between solution and solvent lie in this frequency range. However, data of transverse wave have not been reported, owing to difficulty of measurement. To avoid difficulty in the transverse wave, the reflecting method is used, counting as many pulsed echoes as possible. The method is able to measure the real part of shear impedance, $\rho v_{\mathrm{s}}{ }^{\prime 2}$, where $\rho$ is the density of sample and $v_{\mathrm{s}}{ }^{\prime}$ is the real part of the complex shear velocity. The measured values of $\rho v_{\mathrm{s}}{ }^{\prime 2}$ are compared with Rouse's theory, to test its validity at very high frequencies. The measurement is conducted on toluene solution of polybutene (HV-1900), poly(vinylisobuthylether) (J-30) and polystyrene (PS). The longitudinal velocity and absorption are also measured for $\mathrm{J}-30$, indicating that the ratio of the observed absorption to the classical Stokes-Kirchhoff loss is $20-40$. Since the classical theory considers only the shear viscosity, a total viscous contribution indicating the effects arising from the volume viscosity $\eta_{\mathrm{v}}$ and shear viscosity $\eta_{\mathrm{s}}$ to ultrasonic absorption must be taken into account. In the case of structural relaxation, the ratio $\eta_{\mathrm{v}} / \eta_{\mathrm{s}}$ is essentially temperature independent and rarely higher than 10 or less than 0.5 . The theory has been applied to associated liquids such as water and various alcohols. ${ }^{1}$ On the other hand, in the case of nonassociated liquids, the ratio $\eta_{\mathrm{v}} / \eta_{\mathrm{s}}$ is often very high $(>20)$. The phenomena have been successfully interpreted in terms of the thermal relaxation mechanism, which is due to the time lag for transition of energy from the transitional freedom of molecules to the internal freedom, vibration and rotation. However, there are some difficulties in application of the theory to the present results; 1) the incremental specific heat $\delta C_{\mathrm{p}}$ derived from the measurement is larger than the theoretical value estimated from thermal relaxation mechanism; 2) the thermal relaxation theory is only valid for a pure liquid. Bauer $^{2}$ linearly extrapolated $\delta C_{\mathrm{p}}$ in $5-20 \%$ 
solution into the pure liquid state and compared it with Shottky's function. The consistency has not appeared in the present data obtained by the difference of losses between solution and solvent directly; 3 ) the spectra of the absorption per wavelength are broad, indicating the presence of the cooperative phenomena. ${ }^{4}$ This type of cooperation is in contrast to the thermal relaxation mechanism which should show a single relaxation, ${ }^{3}$ because it arises from the internal freedom of rotation independent of the cooperative motion in the relatively dilute state solution.

Here, a new analysis for the excess absorption is proposed, following concentration fluctuation theory predicted by Romanov and Solov'ev. ${ }^{5}$ This approach affords a better understanding of the observed phenomena.

A necessary consequence of the random motion of the molecules is that the number of molecules per unit volume, in any volume element, is not at every instance equal to its average value but instead fluctuates about this average. The theory of concentration fluctuation in a gas was first developed by Smoluchowski in 1908 and found to be true by the explanation of blue sky with light scattering. It was found that the fluctuation theory held also for colloidal suspensions, for which Svedberg observed particles directly with a microscope in 1912. Vucks and Lisnyanskii postulated that sound absorption of solutions, like light scattering, is related to concentration fluctuations, in 1962. Remanov and Solov'ev calculated the acoustic absorption due to fluctuations, molar enthalpy $H$ and molar volume $V$ to depend on mean fluctuation. When we apply an ultrasonic sound wave to the specimen, adiabatic compression and expansion arise in it. Variation in the pressure $p$ and temperature $T$ in the sound wave alters the magnitude of fluctuation, and a new distribution is established by diffusion. This phenomena is a relaxation process, where the time lag accompanied by diffusion causes sound absorption of solutions.

\section{EXPERIMENTAL}

The reflection method is used to determine the real part of $\rho v_{\mathrm{s}}{ }^{\prime 2}$. A shear impedance cell is similar to the one constructed by Clark and Litovitz. ${ }^{6}$ A 2-cm-diam. 2-cm-long AC-cut crystalline quartz rod was used in frequencies of $8.6,20,60$, and $100 \mathrm{MHz}$. This method, in which real and imaginary part of rigidity cannot be obtained directly, has the advantage of easy treatment. Further, it has no loss of contact between the transducer and quartz rod, so that one can measure over fifty or more echos and determine a low value of $\rho v_{\mathrm{s}}{ }^{2}$ in solution state. Following the theory by Mason $^{7}$ and Piccirelli and Litovitz, ${ }^{4}$ the value $\rho v_{\mathrm{s}}{ }^{\prime 2}$ is expressed by

$$
\rho v_{\mathrm{s}}{ }^{\prime 2}=\left(Z_{\mathrm{q}}{ }^{2} / \rho\right)\left\{\tanh \left[D_{\mathrm{w}}(T) / 40 \text { loge }\right]^{22}\right.
$$

where $\rho$ is the density of sample, $v_{\mathrm{s}}{ }^{\prime}$ is the real part of the complex shear velocity, $Z_{\mathrm{q}}$ $\left(=8.78 \times 10^{5} \mathrm{~g} \mathrm{~cm}^{-2} \mathrm{~s}^{-1}\right)$ is the shear impedance of $\mathrm{AC}$ cut quartz and $D_{\mathrm{w}}(T)$ is the differences between the losses, expressed in decibels per echo, when the quartz is terminated in the liquid and then in air at the same temperature. The amplitude in $\mathrm{db}$ versus number of echo is shown in Figure 1 as an example. The least square fit, indicated in the solid line, determins $D_{\mathrm{w}}(T)$.

The usual interferometric technique ${ }^{8}$ was used to measure the longitudinal wave velocity and absorption at frequencies of 1, 3, 5, 20,60, 100,140 , and $180 \mathrm{MHz}$.

Viscosities are measured by a Cannon Fenske viscometer.

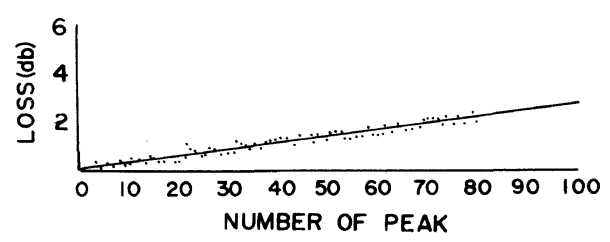

Figure 1. Example of the amplitude in $\mathrm{db}$ versus the number of echos. 
Table I. Molecular weights of samples

\begin{tabular}{lcccc}
\hline \multicolumn{1}{c}{ Sample } & $\bar{M}_{w}$ & $\bar{M}_{w} / \bar{M}_{n}$ & $Z^{\mathrm{a}}$ & $M_{0}{ }^{\mathrm{b}}$ \\
\hline $\begin{array}{l}\text { Polybutene } \\
(\text { HV-1900) }\end{array}$ & 2350 & 2.1 & 42 & 56 \\
$\begin{array}{l}\text { Poly(vinylisobutylether) } \\
(\text { J-30) }\end{array}$ & 5700 & 2.2 & 57 & 100 \\
$\begin{array}{l}\text { Polystyrene } \\
\text { (P.S.) }\end{array}$ & 10000 & 1.06 & 95 & 105 \\
\hline
\end{tabular}

a $Z$, degree of polymerization; $N q, q=5$.

b $M_{0}$, molecular weight of monomer.

Volume expansion coefficient is measured by a picnometer modified by the author.

The molecular characteristics of the samples are listed in Table I. The distribution curves of molecular weight of HV-1900 and J-30 are obtained by gel permeation chromatograph.

\section{RESULTS AND ANALYSIS}

\section{a) Shear Moduli}

The values of $\rho v_{\mathrm{s}}{ }^{2}$ versus frequency are shown in Figure 2. For the monodisperse in case of PS, the theoretical curve is derived from Rouse's theory, ${ }^{9}$ in which real and imaginary parts of rigidity are, respectively, represented by

$$
\begin{aligned}
G_{1} & =\frac{C R T}{M} \sum_{p=1}^{N} \frac{\left(\omega \tau_{\mathrm{p}}\right)^{2}}{1+\left(\omega \tau_{\mathrm{p}}\right)^{2}}, \\
G_{2} & =\frac{C R T}{M} \sum_{p=1}^{N} \frac{\omega \tau_{\mathrm{p}}}{1+\left(\omega \tau_{\mathrm{p}}\right)^{2}}+\omega \eta_{\mathrm{s}},
\end{aligned}
$$

where $C$ is the concentration $\left(\mathrm{g} \mathrm{cm}^{-3}\right), R$ is the gas constant, $T$ is the absolute temperature, $M$ is the molecular weight, $\omega$ is the angular frequency (radian), $\chi_{p}$ is the relaxation time of the $p$ th mode of motion, and $\eta_{\mathrm{s}}$ is the viscosity of solvent. The value of $\tau_{p}$ is given by

$$
\tau_{\mathrm{p}}=\frac{1}{\sin ^{2}\left[\frac{p \pi}{2(N+1)}\right]} \frac{M\left(\eta-\eta_{\mathrm{s}}\right)}{\operatorname{CRT} \sum_{p=1}^{N} \frac{1}{\sin ^{2}[p \pi / 2(N+1)]}}
$$

where $p=1,2, \cdots, N, N$ is the number of segment, $\eta$ is the static viscosity of solution, and $\eta-\eta_{\mathrm{s}}$ is the contribution of polymer to the viscosity of the solution in steady flow. When the Rouse mode number $N$ is large,

$$
\begin{aligned}
\tau_{\mathrm{p}} & =\frac{6}{\pi^{2} p^{2}} \frac{M\left(\eta-\eta_{\mathrm{s}}\right)}{C R T}, \\
\tau_{1} & =\frac{6}{\pi^{2}} \frac{M\left(\eta-\eta_{\mathrm{s}}\right)}{C R T} .
\end{aligned}
$$

For HV-1900 and J-30 with a most probable distribution of molecular weights $\left(\bar{M}_{w} / \bar{M}_{n}=2\right)$, the following relations ${ }^{10}$ are used.

$$
G_{1}=\frac{C R T}{M} \sum_{p=1}^{N} \int_{0}^{\infty} \frac{\left(\omega \tau_{1}\right)^{2} \mu^{4} / p^{4}}{1+\left(\omega \tau_{1}\right)^{2} \mu^{4} / p^{4}} \exp (-\mu) \mathrm{d} \mu
$$

$$
\begin{array}{r}
G_{2}=\frac{C R T}{M} \sum_{p=1}^{N} \int_{0}^{\infty} \frac{\omega \tau_{1} \mu^{2} / p^{2}}{1+\left(\omega \tau_{1}\right)^{2} \mu^{4} / p^{4}} \\
\exp (-\mu) \mathrm{d} \mu+\omega \eta_{\mathrm{s}},
\end{array}
$$

where $\mu=M / \bar{M}_{n}$ and $\tau_{1}=3 \eta \bar{M}_{n} /\left(\pi^{2} C R T\right)$. The Rouse mode number $N$ is given by the relation $N q=Z$, where $q$ is the number of monomer units in a segment and $Z\left(=M / M_{0}\right)$ is the degree of polymerization. The values of $G_{1}$ and $G_{2}$ are estimated from $N=11$ and $q=5$ in eq 7 and 8 . The value of $N$ in the eq $2,3,7$, and 8 is estimated as 11 , with $q=5$ as obtained from the results of oligostyrene and oligobutene in previous papers. ${ }^{11,12}$ Knowing $G_{1}$ and $G_{2}$, $\rho v_{\mathrm{s}}{ }^{\prime 2}$ is calculated by

$$
\rho v_{\mathrm{s}}{ }^{\prime 2}=\frac{G_{1}}{2}\left\{1+\left[1+\left(\frac{G_{2}}{G_{1}}\right)^{2}\right]^{1 / 2}\right\} .
$$

In Figure 2, the solid lines show the composit values of eq 2 and 3; the dotted lines indicate those of eq 7 and 8 . The difference between mono and poly disperse systems was found to have little effect on the value of $\rho v_{\mathrm{s}}{ }^{\prime 2}$ in this high frequency region with respect to a dilute solution. Regardless of differencies in molecular weights ( $c f$., Figure $2 \mathrm{a}$ and $2 \mathrm{~b}$ ), concentration ( $c f$., Figure $2 \mathrm{~b}$ and $2 \mathrm{c}$ ), temperature ( $c f$., Figures $2 \mathrm{~b}$ and $2 \mathrm{e}$ ), and distribution of 
HV- 1900 , toluene $5 \mathrm{~g} / 100 \mathrm{ml} 23^{\circ} \mathrm{C}$

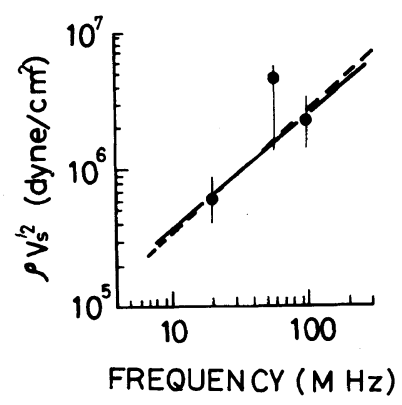

(a)

$\mathrm{J}-30$, toluene $5 \mathrm{~g} / 100 \mathrm{ml} 23^{\circ} \mathrm{C}$

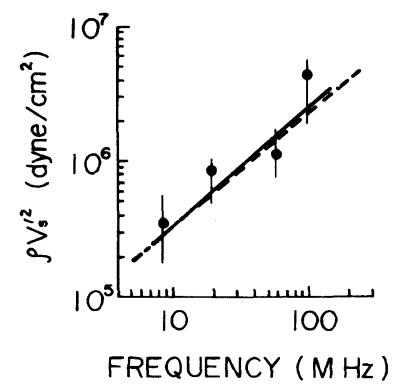

(b)

$\mathrm{J}-30$, toluene $10 \mathrm{~g} / 100 \mathrm{ml} 23^{\circ} \mathrm{C}$

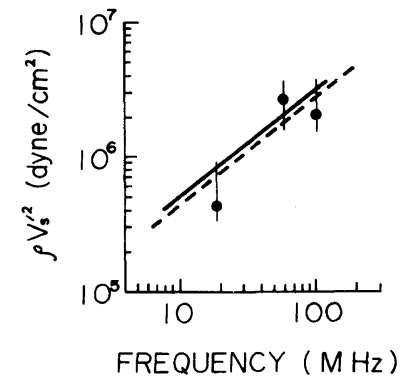

(c)

P.S. $(10,000)$, toluene $5 \mathrm{~g} / 100 \mathrm{ml} 23^{\circ} \mathrm{C}$

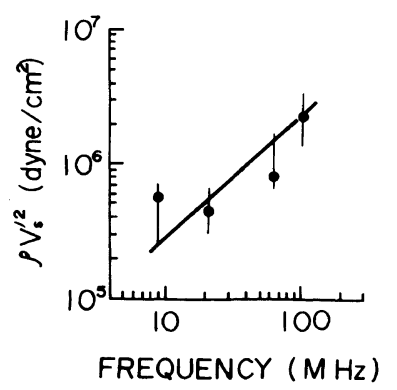

(d)
$\mathrm{J}-30$, toluene $5 \mathrm{~g} / 100 \mathrm{ml} 3^{\circ} \mathrm{C}$

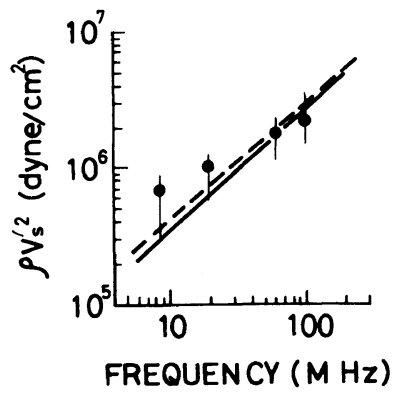

(e)

Figure 2. The values of $\rho v_{\mathrm{s}}{ }^{\prime 2}$ versus frequency of (a) $\mathrm{HV}-1900$, toluene $(5 \mathrm{~g} / 100 \mathrm{ml})$ at $23^{\circ} \mathrm{C}$, (b) $\mathrm{J}-30$, toluene $(5 \mathrm{~g} / 100 \mathrm{ml})$ at $23^{\circ} \mathrm{C}$, (c) $\mathrm{J}-30$, toluene $(10 \mathrm{~g} / 100 \mathrm{ml})$ at $23^{\circ} \mathrm{C}$, (d) P.S. $(10,000)$, toluene $(5 \mathrm{~g} / 100 \mathrm{ml})$ at $23^{\circ} \mathrm{C}$, (e) $\mathrm{J}-30$, toluene $(5 \mathrm{~g} / 100 \mathrm{ml})$ at $3^{\circ} \mathrm{C}$. The top and bottom of error bar show the maximum and minimum of measured values. The dots show mean values.

molecular weight ( $c f$., Figures $2 \mathrm{~b}$ and $2 \mathrm{~d}$ ), the measured values of $\rho v_{\mathrm{s}}{ }^{2}$ match the theoretical values. This consistency enables us to estimate the values of $G_{1}$ and $G_{2}$ by knowing only temperature, concentration and molecular weights of the samples.

\section{b) Longitudinal Moduli}

The complex bulk modulus is written as $K=$ $K_{1}+i K_{2}=K_{1}+i \omega \zeta^{\prime}$ and the complex shear modulus $G^{*}=G_{1}+G_{2}=G_{1}+i \omega \eta^{\prime}$, where $\zeta^{\prime}$ is dynamic volume viscosity and $\eta^{\prime}$, the dynamic shear viscosity. If the complex longitudinal wave modulus is written as $M^{*}=M_{1}+i M_{2}$, the relation among the velocity of longitudinal waves $v_{1}$, the attenuation of longitudinal waves $\alpha_{1}$ in neper $\mathrm{cm}^{-1}$ and the modulus can be shown as follows

$$
\begin{aligned}
& M_{1}=\rho v_{1}^{2} \frac{1-\left(\alpha_{1} v_{1} / \omega\right)^{2}}{\left(1+\alpha_{1}{ }^{2} v_{1}{ }^{2} / \omega^{2}\right)^{2}}=K_{1}+\frac{4}{3} G_{1}, \\
& M_{2}=\rho v_{1}^{2} \frac{2 \alpha_{1} v_{1} / \omega}{\left(1+\alpha_{1}{ }^{2} v_{1}{ }^{2} / \omega^{2}\right)^{2}}=K_{2}+\frac{4}{3} G_{2} .
\end{aligned}
$$

Using eq 11 , the following equation is obtained for $\alpha_{1} v_{1} / \omega \ll 1$, 


$$
\frac{\alpha_{1}}{f^{2}}=\frac{2 \pi^{2}}{\rho v_{1}^{3}}\left(\zeta^{\prime}+\frac{4}{3} \eta^{\prime}\right) .
$$

If the differences between solution and solvent in absorption of longitudinal waves, dynamic volume and shear viscosities are written as $\Delta \alpha_{1}, \Delta \zeta^{\prime}$, and $\Delta \eta^{\prime}$, respectively, the following equation is obtained;

$$
\frac{\Delta \alpha_{1}}{f^{2}}=\frac{2 \pi^{2}}{\rho v_{1}^{3}}\left(\Delta \zeta^{\prime}+\frac{4}{3} \Delta \eta^{\prime}\right) .
$$

The difference in shear, $\Delta \alpha_{\mathrm{s}}$ is derived from the second term of eq 13 and given by

$$
\frac{\Delta \alpha_{\mathrm{s}}}{f^{2}}=\frac{8 \pi^{2}}{3 \rho v_{1}^{3}} \Delta \eta^{\prime}
$$

where the value of $\Delta \eta^{\prime}$ can be determined by estimation of the first term in the right side of eq 8 which should be $\omega \Delta \eta^{\prime}$. The values of $\tau_{1}$ are obtained from eq 6 and shown in Table II. The value of $\Delta \alpha_{\mathrm{s}} / f^{2}$ can be calculated from eq 14 in which $\Delta \eta^{\prime}$ is replaced by the factor $\left(G_{2}-\omega \eta_{\mathrm{s}}\right) / \omega$ and the results are shown by the solid line in Figure 3 and Table II. Then $\Delta \zeta^{\prime}$ in Table II is calculated from eq 13 and 14 .

\section{DISCUSSION}

It is seen in Figure 3 and Table II that the experimental values of longitudinal excess absorption $\Delta \alpha_{1}$ are much larger than those of shear absorption $\Delta \alpha_{\mathrm{s}}$ with a ratio of $\Delta \zeta^{\prime} / \Delta \eta>20$. At first sight, the phenomena with high value of $\Delta \zeta^{\prime} / \Delta \eta$ seem to be due to a thermal relaxation mechanism. However, there are three reasons why this mechanism is not adequate to explain the results; First, thermal relaxation theory ${ }^{13}$ proposes that $\delta C_{\mathrm{p}}$ as referring to one mole can be written as

$$
\delta C_{\mathrm{p}}=\frac{2 \Delta \mu_{\max }}{\pi} \frac{C_{\mathrm{p}}}{\gamma-1},
$$

where $\Delta \mu_{\max }$ is the maximum value of absorption per wave length $\Delta \mu(=\Delta \alpha \hat{\lambda}), \gamma$ is $C_{\mathrm{p}} / C_{\mathrm{v}}$ and $C_{\mathrm{p}}$ and $C_{\mathrm{v}}$ are specific heats at constant pressure and volume, respectively. Using the
$\mathrm{J}-30$, toluene $5 \mathrm{~g} / 100 \mathrm{ml}$

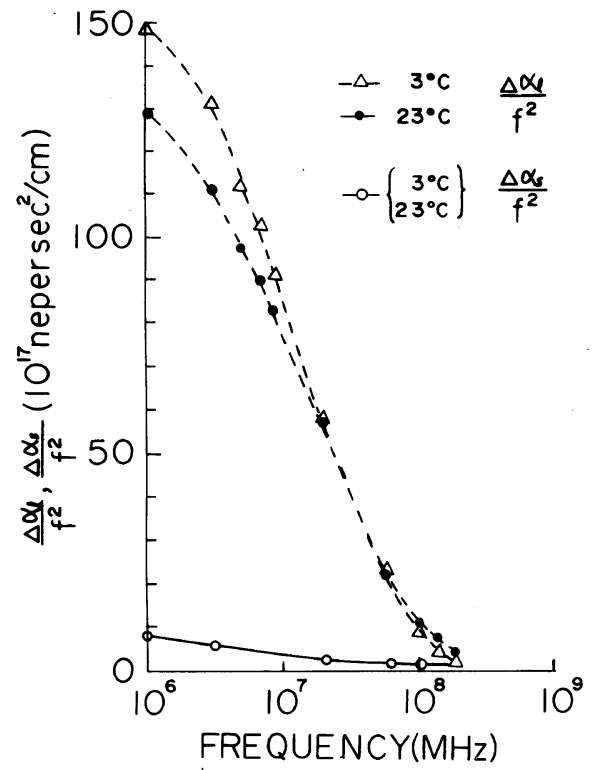

Figure 3. Values of $\Delta \alpha_{1} / f^{2}$ and $\Delta \alpha_{s} / f^{2}$ versus frequency. The dots and triangles are measured values. The solid line and circles are theoretical values by Lamb's theory.

values of $v_{1}, C_{\mathrm{p}}, \mu_{\max }$, and coefficient of expansion $\theta, \delta C_{\mathrm{p}}$ is obtained as shown in Table III, where $\gamma-1$ is calculated from the relation $v_{1}{ }^{2}=\left(\gamma^{-1}\right) J C_{\mathrm{p}} / \theta^{2} T{ }^{14}$ where $J=4.187 \mathrm{~J} \mathrm{cal}^{-1}$. On the other hand Shottky introduced the contribution to specific heat due to a doubly excited energy state above a nondegenerated ground state, giving

$$
\delta C_{\mathrm{p}}=R\left[\frac{\Delta H^{0}}{R T}\right]^{2} \frac{2 \exp \left(-\Delta H^{0} / R T\right)}{\left[1+2 \exp \left(-\Delta H^{0} / R T\right)\right]^{2}},
$$

where $\Delta H^{0}$ is the difference in enthalpy between ground and excited states. The form of eq 16 versus $\Delta H^{0} / R T$ has been given by Litovitz, ${ }^{6}$ showing that the maximum value of $\delta C_{\mathrm{p}}$ occurring at $\Delta H=2.4 R T$ is 1.52 cal mol ${ }^{-1}{ }^{\circ} \mathrm{C}^{-1}$. Comparing the results in Table III, it is clear that the difference between them is far beyond experimental error. Next, the validity of eq 16 is only for the case of unimolecular reaction. ${ }^{13}$ Its application to the 
Table II. Acoustical quantities of J-30, toluene mixture

\begin{tabular}{|c|c|c|c|c|c|c|}
\hline Temp & & Frequency & $\Delta \alpha_{\mathrm{s}} / f^{2}$ & $\Delta \alpha_{1} / f^{2}$ & $\Delta \zeta^{\prime}$ & \\
\hline${ }^{\circ} \mathrm{C}$ & & $\mathrm{MHz}$ & $\begin{array}{l}\mathrm{s}^{2} \mathrm{~cm}^{-1} \\
\times 10^{-17}\end{array}$ & $\begin{array}{l}\mathrm{s}^{2} \mathrm{~cm}^{-1} \\
\times 10^{-17}\end{array}$ & $\begin{array}{c}\mathrm{gcm}^{-1} \mathrm{~s}^{-1} \\
\times 10^{-3}\end{array}$ & \\
\hline \multirow[t]{10}{*}{3} & $\rho=0.8825\left(\mathrm{~g} \mathrm{~cm}^{-3}\right)$ & 1 & 7.6 & 148 & 183 & 24.6 \\
\hline & $v_{1}=1.402 \times 10^{5}\left(\mathrm{~cm} \mathrm{~s}^{-1}\right)$ & 3 & 5.3 & 131 & 155 & 31.6 \\
\hline & $\tau_{1}=1.59 \times 10^{-8}(1 / \mathrm{s})$ & 5 & 3.9 & 114 & 136 & 37.8 \\
\hline & $\eta=1.291 \times 10^{-2}$ (poise) & 7 & 3.4 & 105 & 125 & 39.8 \\
\hline & $\eta_{\mathrm{s}}=7.77 \times 10^{-3}$ (poise) & 9 & 2.9 & 92.0 & 110 & 41.0 \\
\hline & $G / C R T=2.01 \times 10^{5}$ & 20 & 1.5 & 57.6 & 68.4 & 34.4 \\
\hline & $N=11$ & 60 & 0.99 & 16.7 & 19.8 & 21.2 \\
\hline & & 100 & 0.67 & 7.62 & 8.56 & 13.8 \\
\hline & & 140 & 0.50 & 4.11 & 4.45 & 9.63 \\
\hline & & 180 & 0.41 & 2.82 & 2.97 & 7.84 \\
\hline \multirow[t]{10}{*}{23} & $\rho=0.8630\left(\mathrm{~g} \mathrm{~cm}^{-3}\right)$ & 1 & 7.5 & 128 & 120 & 21.4 \\
\hline & $v_{\mathrm{d}}=1.313 \times 10^{5}\left(\mathrm{~cm} \mathrm{~s}^{-1}\right)$ & 3 & 5.4 & 111 & 105 & 26.1 \\
\hline & $\tau_{1}=1.06 \times 10^{-8}(1 / \mathrm{s})$ & 5 & 4.3 & 97.1 & 91.8 & 28.7 \\
\hline & $\eta=9.73 \times 10^{-3}$ (poise) & 7 & 3.8 & 89.4 & 84.7 & 30.0 \\
\hline & $\eta_{\mathrm{s}}=6.02 \times 10^{-3}$ (poise) & 9 & 3.5 & 83.8 & 79.5 & 30.7 \\
\hline & $G / C R T=2.16 \times 10^{5}$ & 20 & 2.3 & 59.5 & 56.7 & 33.3 \\
\hline & $N=11$ & 60 & 1.2 & 21.8 & 20.5 & 23.8 \\
\hline & & 100 & 0.80 & 10.8 & 9.90 & 16.7 \\
\hline & & 140 & 0.61 & 6.52 & 5.85 & 12.9 \\
\hline & & 180 & 0.50 & 4.27 & 3.73 & 10.0 \\
\hline
\end{tabular}

Table III. Estimation of $\delta C_{\mathrm{p}}$ derived from experimental values

\begin{tabular}{|c|c|c|c|c|}
\hline Temp & $C_{\mathbf{p}}$ & \multirow{2}{*}{$\gamma-1^{\mathrm{a}}$} & \multirow{2}{*}{$\Delta \mu_{\max }$} & \multirow{2}{*}{ 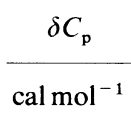 } \\
\hline${ }^{\circ} \mathrm{C}$ & $\operatorname{erg} g^{-1}$ & & & \\
\hline 3 & $1.63 \times 10^{7}$ & 0.374 & $1.7 \times 10^{-3}$ & 2.13 \\
\hline---1 & ----- & --- & ----- & ----- \\
\hline 23 & $1.68 \times 10^{7}$ & 0.341 & $1.9 \times 10^{-3}$ & 2.34 \\
\hline
\end{tabular}

a $\gamma-1$ was calculated from the relation $v_{1}{ }^{2}=(\gamma-1) J C_{\mathrm{p}} /$ $\theta^{2} T^{14}$, where $\theta=1.06 \times 10^{-3}$.

solution is doubtful. The third weak point is the fact that the thermal relaxation has difficulty in explaining the broadness of the spectrum of $\Delta \mu$. Figure 4 shows the curve of $\Delta \mu$ versus frequency where the dots and triangles are experimental values and the solid line is a single relaxation curve characteristic of the thermal relaxation and clearly deviates from the experimentals. Thus neither structural re- laxation nor thermalrelaxation can account for the excess absorption data.

The most probable interpretation should be a concentration fluctuation mechanism predicted by Romanov and Solov'ev, ${ }^{5}$ because the present system is a mixture. They attribute the excess absorption to fluctuation which is treated as regions of variable concentration in the volume devided into elements $\mathrm{d} V$, and assume that the resorption of the fluctuation proceeds according to the diffusion equation $\partial x / \partial t=$ $D \nabla^{2} x$, where $x$ is the concentration in the volume $\mathrm{d} V$ and $D$ is diffusion constant. The amplitudes involved in expansion into the Fourier spectrum of concentration fluctuation $B_{\mathrm{f}}$ vary according to the equation,

$$
\frac{\partial B_{\mathrm{f}}}{\partial t}=-\frac{1}{\tau_{\mathrm{f}}}\left(\bar{B}_{\mathrm{f}}-B_{\mathrm{f}}\right),
$$

where $\bar{B}_{\mathrm{f}}$ is the average value of $B_{\mathrm{f}}$ for given $p$ and $T, f$ is the wave number of the Fourier 


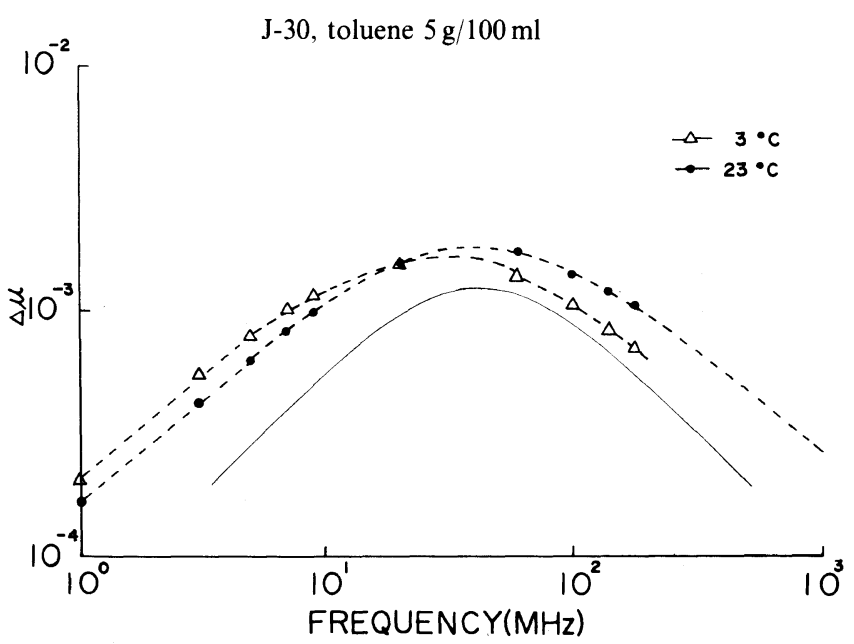

Figure 4. Curve of $\Delta \mu(=\Delta \alpha \lambda)$ versus frequency. The dots and triangles are measured values. The solid line is the theoretical value of a single relaxation and its maximum frequency is plotted in the same manner as that of the measured curve on $23^{\circ} \mathrm{C}$.

spectral expansion, and $\tau_{\mathrm{f}}$ is relaxation time and defined as

$$
\tau_{\mathrm{f}}=1 / D f^{2} \text {. }
$$

The excess volume viscosity coefficient $\Delta \zeta^{\prime \prime}$ should be presented by the following equations,

$$
\begin{aligned}
& \Delta \zeta^{\prime}=A K_{\infty}^{2} T \frac{f_{\mathrm{m}}}{D} L\left(\frac{\omega}{D f_{\mathrm{m}}^{2}}\right), \\
& L\left(\frac{\omega}{D f_{\mathrm{m}}{ }^{2}}\right)=\frac{1}{f_{\mathrm{m}} \sqrt{D / \omega}} \int_{0}^{f_{\mathrm{m} \sqrt{D / \omega}}} \frac{x^{4}}{1+x^{4}} \mathrm{~d} x, \\
& A=\frac{V^{2} k}{4 \pi^{2} \psi^{2}}\left(\frac{v}{V}-\frac{a_{\infty}}{C_{\mathrm{p} \infty}} h\right)^{2},
\end{aligned}
$$

where $K_{\infty}$ is the instantaneous adiabatic modulus, $V$ is molar volume, $k$ is Boltzman constant, $\psi=\partial^{2} \Phi_{0} / \partial c^{2}$ where $\Phi_{0}$ is the molar thermodynamic potential in the absence of fluctuation, $v=\partial^{2} V_{0} / \partial c^{2}$ where $V_{0}$ is the molar volume in the absence of fluctuation, $a_{\infty}$ is instantaneous molar heat capacity, $C_{\mathrm{p} \infty}$ is instantaneous molar heat capacity, $h=$ $\partial^{2} H_{0} / \partial c^{2}$ where $H_{0}$ is molar enthalpy in the absence of fluctuation, and $f_{\mathrm{m}}$ is the maximum wave number of Fourier spectral expansion of fluctuation. The value of $A$ involves a large amount of uncertainty, ${ }^{15}$ so an explicit calculation was not given. This term can be considered a constant with temperature. ${ }^{5}$ Taking into account the smallness of $G_{1}$ and the difference between $K_{\infty}$ and $K_{0}$ which is the equilibrium modulus, the temperature dependence of $K_{\infty}$ should be indentified with $M_{1}$.

In dilute solutions of linear polymer, the molecular motions are non-cooperative and hence segments can freely rotate. An individual molecule forms a random coil, the most probable shape on the basis of statistical theory. The mean square end-to-end distance of molecule $L^{2}$ is represented by

$$
L^{2}=N a^{2},
$$

where $N$ is the total number of segments, and $a$ is the length of a segment. When isolated polymer molecules move through the surrounding fluid unperturbed by the movement of other polymer molecules, $D$ is represented by the Einstein relation, ${ }^{16}$

$$
D=k T / N f^{\prime},
$$

where $f^{\prime}$ is a submolecule friction constant and $N f^{\prime}$, a molar friction constant.

One may deduce a relation between $D$ and $\eta-\eta_{\mathrm{s}}$. According to Debye, ${ }^{17}$ the value of 
$\eta-\eta_{\mathrm{s}}$ is given by

$$
\eta-\eta_{\mathrm{s}}=\frac{n L^{2} N f^{\prime}}{36}
$$

where $n$ is the number of molecules per unit volume of solution. Eliminating $N f^{\prime}$ from eq 23 and 24 , one obtains

$$
D\left(\eta-\eta_{\mathrm{s}}\right)=\frac{k \operatorname{Tn} L^{2}}{36}
$$

Putting $n=0.05\left(\mathrm{~g} \mathrm{~cm}^{-3}\right) 6.03 \times 10^{23} / 5700(\mathrm{~g})=$ $5.29 \times 10^{18} \times 1 \mathrm{~cm}^{-3}, \quad a=4 \times 1.5 \times 10^{-8} \times 5 \mathrm{~cm}$ and the values of $\eta, \eta_{\mathrm{s}}$, and $N$ given in Table II into eq 22 and 25 , one obtains the value of $D$ given in Table IV.

First, the volume viscosities $\Delta \zeta^{\prime}$ are plotted versus frequency. Comparing the plots with the theoretical value of $L\left(\omega / D f_{\mathrm{m}}{ }^{2}\right)$ versus frequency, $f_{\mathrm{m}}$ can be determined from $\omega / D f_{\mathrm{m}}{ }^{2}=1$ on the horizontal axis. The results are shown in Table IV which indicates that $f_{\mathrm{m}}$ is constant with temperature. Secondly, the values of $\Delta \zeta^{\prime}$ | $\left(K_{\infty}{ }^{2} T f_{\mathrm{m}} / D\right)$ versus $\omega / D f_{\mathrm{m}}{ }^{2}$ are plotted as shown in Figure 5, where the values of $L\left(\omega / D f_{\mathrm{m}}{ }^{2}\right)$ are also plotted against $\omega / D f_{\mathrm{m}}{ }^{2}$. The value of $A$ is determined as the value of the vertical axis of $\Delta \zeta^{\prime} /\left(K_{\infty}{ }^{2} T f_{\mathrm{m}} / D\right)$ which corresponds to the unit value of $L\left(\omega / D f_{\mathrm{m}}{ }^{2}\right)$. The values of $\Delta \zeta^{\prime \prime}$ reduced by $K_{\infty}{ }^{2} T f_{\mathrm{m}} / D$ fit very well with the values of $L\left(\omega / D f_{\mathrm{m}}{ }^{2}\right)$ at both $3^{\circ} \mathrm{C}$ and $23^{\circ} \mathrm{C}$.

The value of $f_{\mathrm{m}}$ defines the limit of applicability of the model proposed by Romanov and Solov'ev and the validity of $f_{\mathrm{m}} \sim 10^{7} \mathrm{~cm}^{-1}$ is supported by them. If a molecule in a solvent is like a rod, it should be assumed that $f_{\mathrm{m}} \sim$ $1 / L$. Putting the value of $N=11$ and $a=4 \times 1.5 \times 10^{-8} \times 5 \mathrm{~cm}$ into eq $22,1 / L$ is estimated as $1.01 \times 10^{6} \mathrm{~cm}^{-1}$. The mean length between molecules $r$ is related to $1 / r \sim\left(N_{\mathrm{A}} / V\right)^{1 / 3}, \quad$ where $N_{\mathrm{A}}$ is Avogadro's number. Since $N_{\mathrm{A}}=6.02 \times 10^{23}, \quad V=100$. $\left(\mathrm{cm}^{3}\right) \times 5700 / 5,1 / r$ is nearly equal to $1.74 \times$ $10^{6} \mathrm{~cm}^{-1}$. These values give the relation $f_{\mathrm{m}}>$ $1 / r>1 / L$, which suggests the shapè of molecule in a solvent to be more like a sphere
Table IV. Values of $D$ and $f_{\mathrm{m}}$

\begin{tabular}{cccccc}
\hline Temp & $D$ & & $f_{\mathrm{m}}$ & $\tau_{\mathrm{fm}}$ & $\tau_{1} / \tau_{\mathrm{fm}}$ \\
& $\frac{{ }^{\circ} \mathrm{C}}{\mathrm{cm}^{2} \mathrm{~s}^{-1}}$ & $\mathrm{~cm}^{-1}$ & $\mathrm{~s}$ & \\
\hline 3 & $8.09 \times 10^{-7}$ & $1.91 \times 10^{7}$ & $3.38 \times 10^{-9}$ & 4.70 \\
\hline 23 & $1.20 \times 10^{-6}$ & $1.90 \times 10^{7}$ & $2.31 \times 10^{-9}$ & 4.59 \\
\hline
\end{tabular}

$\mathrm{J}-30$, toluene $5 \mathrm{~g} / 100 \mathrm{ml}$

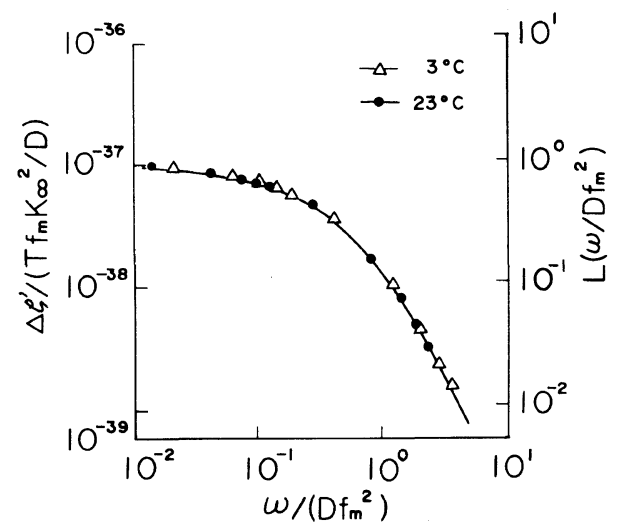

Figure 5. Plots of $\Delta \zeta^{\prime} /\left(K_{\infty}{ }^{2} T f_{\mathrm{m}} / D\right)$ and $L\left(\omega / D f_{\mathrm{m}}{ }^{2}\right) v e$ rsus $\omega / D f_{\mathrm{m}}{ }^{2}$. The dots and triangles are measured values. The solid line is the theoretical value of $L\left(\omega / D f_{\mathrm{m}}{ }^{2}\right)$.

than a rod.

The relaxation time of concentration fluctuation, $\tau_{\mathrm{f}}$ can be estimated by eq 18 and the value of $f_{\mathrm{m}}$, as shown in Table IV. Comparing $\tau_{\mathrm{f}}$ with the relaxation time of Rouse mode motion $\tau_{1}$, the ratios of $\tau_{1} / \tau_{\mathrm{f}}$ are 4.70 and 4.59 for $3{ }^{\circ} \mathrm{C}$ and $23^{\circ} \mathrm{C}$, respectively. On the basis of eq 5 and 6 , this means that the minimum relaxation time of fluctuation corresponds to the second mode motion of the Rouse mode. Considering $\tau_{1}$ has the order of time necessary for movement of whole one molecule in shear deformation, about half the length of one molecule is the minimum size of fluctuation, where there is one or no molecule. This is very reasonable for all points of the fluctuation theory. 


\section{CONCLUSIONS}

Shear moduli were measured for some polymer toluene solutions, and the results had good agreements with those expected from Rouse's and Lamb's theory. It was found that the concentration fluctuation theory by Romanov-Solovev explains the acoustic excess absorption of poly(vinylisobutylether) solution in toluene very well.

Acknowledgment. The author wishes to thank Mr. Yoshida of Shimadzu Co., Ltd. for the measurements of molecular weight distribution of HV-1900 and J-30 using GPC.

\section{REFERENCES}

1. N. Hirai and H. Eyring, J. Appl. Phys., 29, 810 (1958).

2. H. J. Bauer and H.. Hässler, The 6th International Congress on Acoustics, J-69.

3. H. Nomura, S. Kato, and Y. Miyahara, Nihon
Kagaku Zasshi, 90, 40 (1969); ibid., 91, 837 (1970).

4. R. Piccirerei and T. A. Litovitz, J. Acoust. Soc. Am., 29, 1009 (1957).

5. V. P. Romanov and V. A. Solov'ev, Soviet PhysicsAcoustics, 11, 68 (1965).

6. A. E. Clark and T. A. Litovitz, J. Acoust. Soc. Am., 32, 1221 (1960).

7. W. P. Mason, Phys. Rev., 73, 1074 (1948).

8. for instance, Heuter and Bolt, "Sonics," John Wiley \& Sons, New York, N. Y., 1965, p 338.

9. P. E. Rouse, J. Chem. Phys., 21, 1272 (1953).

10. A. J. Barlow, G. Harrison, and J. Lamb, Proc. $R$. Soc. A, 282, 228 (1964).

11. R. Kono and H. Yoshizaki, J. Appl. Phys., 47, 5319 (1976).

12. H. Yoshizaki and R. Kono, Acoustica, 37, 326 (1977).

13. J. Lamb, in "Physical Acoustics," Vol. II, W. P. Mason, Ed., Academic Press, New York, N. Y., 1965, Part A, p 222.

14. J. Lamb, in "Physical Acoustics," Vol. II, W. P. Mason, Ed., Academic Press, New York, N. Y., 1965, Part A, p 224.

15. V. A. Solovyev, C. J. Montrose, M. H. Watkins, and T. A. Litovitz, J. Chem. Phys., 48, 2155 (1968).

16. F. Bueche, J. Chem. Phys., 20, 1959 (1952).

17. P. Debye, J. Chem. Phys., 14, 636 (1946). 